\title{
ESTUDO RETROSPECTIVO DE DEZ CASOS DE PERITONITE INFECCIOSA FELINA DIAGNOSTICADOS ENTRE 2000-2013 NA REGIÃO SUL DO RIO GRANDE DO SUL
}

Recebido: 29/06/2015

SAPIN, Carolina da Fonseca "; SILVA-MARIANO, Luisa Cerqueira ";

FIALHO-XAVIER, Aline Galiza ${ }^{2}$; GRECCO-CORRÊA, Luisa ${ }^{2}$;

BASSI, Jordana Nunes ${ }^{2}$; SCHEID, Haide Valeska ${ }^{3}$; GRECCO, Fabiane Borelli ${ }^{4}$.

Aceito: 10/05/2016

${ }^{1}$ Programa de Pós-Graduação em Veterinária, UFPEL; ${ }^{2}$ Graduação em Veterinária/UFPEL; ${ }^{3}$ Programa de Residência em área Profissional de Saúde, Faculdade de Veterinária/UFPEL; ${ }^{4}$ Professora Adjunta, Doutora, Departamento de Patologia Animal, Faculdade de Veterinária, UFPEL.

\section{RESUMO}

$\mathrm{P}$ eritonite Infecciosa Felina (PIF) é uma doença viral imunomediada, caracterizada por serosite e vasculite fibrinosas disseminadas. $O$ objetivo deste trabalho foi descrever um estudo de dez casos da doença, diagnosticados entre 2000-2013 na região sul do Rio Grande do Sul. Neste período foram analisados materiais de 318 felinos, dos quais, dez casos $(3,14 \%)$ tiveram diagnóstico de PIF. Destes casos, $70 \%$ dos animais apresentaram a forma efusiva da enfermidade e $30 \%$ a forma não-efusiva. Não houve preponderância em relação a raça dos animais afetados. A idade dos felinos variou entre seis meses e quatro anos. A evolução clínica da doença até a morte esteve entre 7 e 45 dias e os sinais clínicos encontrados foram: anorexia, emagrecimento, icterícia, ascite, vômitos, anemia e hálito amoniacal. As lesões macroscópicas observadas foram variáveis e incluíam peritonite e focos esbranquiçados sobre as serosas de diversas vísceras nas duas formas de apresentação da doença. Histologicamente, em ambas formas diagnosticadas, havia vasculite e perivasculite granulomatosas ou piogranulomatosas em diversos órgãos. O diagnóstico nos casos analisados foi realizado com base na história clínica, achados de necropsia e histopatologia. Conclui-se que a Peritonite Infecciosa Felina ocorre na zona de abrangência do Laboratório Regional de Diagnóstico, na região sul do Rio Grande do Sul, porém o número real de animais afetados pode ser maior que o descrito neste trabalho, em virtude da confirmação do diagnóstico por necropsia ou histopatologia não ser realizado em todos os casos suspeitos.

Palavras-chave: Gatos. Coronavírus. Peritonite. 


\section{INTRODUÇÃO}

Os felídeos, e dentre eles, principalmente, os felinos domésticos, são suscetíveis às infecções por coronavírus (DRECHSLER et al., 2011; GELBERG, 2013). Nos gatos, as cepas de coronavírus têm um amplo espectro de virulência, causando desde enterites de gravidade variável até uma doença sistêmica denominada de Peritonite Infecciosa Felina (PIF) (DRECHSLER et al., 2011). Esta é uma doença viral imunomediada, progressiva e que, com raras exceções, é fatal dentro de poucas semanas (BRAUND, 1994). A patogênese da doença é complexa, com imunidade celular desempenhando um papel protetor, enquanto que a resposta imune humoral é geralmente prejudicial, colaborando com a disseminação viral por opsonização e por resultar na formação de imunocomplexos (ADDIE et al., 2009). Os imunocomplexos são depositados em pequenos vasos sangüíneos, onde se fixam e ativam o complemento, sendo fagocitados por macrófagos (GELBERG, 2013; HOSKINS; LOAR, 1993). Macrófagos infectados, então, promovem reações teciduais imunomediadas e um ciclo vicioso de dano vascular mediado por complemento acontece, gerando componentes quimiotáticos e atraindo neutrófilos, os quais liberam enzimas proteolíticas, causando mais dano tecidual (KIPAR et al., 2005). Essas reações podem ser vistas como alterações degenerativas, proliferativas e inflamatórias em paredes de vasos sangüíneos, particularmente nas camadas endoteliais e mediais de pequenas veias e artérias, nas serosas peritoneal, pleural e no tecido conjuntivo intersticial de órgãos parenquimatosos (ADDIE et al., 2009; DRECHSLER et al., 2011; HOSKINS; LOAR, 1993).

Felinos de todas as idades são suscetíveis à infecção, porém são afetados, com maior frequência, animais entre seis meses e cinco anos de idade (ADDIE et al. 2009; GELBERG, 2013). O vírus é encontrado nas secreções e excreções dos animais infectados, que durante a fase aguda da doença o transmitem via fecal-oral, oral-oral e oral-nasal. É provável que portadores assintomáticos também transmitam o vírus através das fezes, e menos comumente pela urina ou secreções oro-nasais (OLIVEIRA et al., 2003). Outros fatores que podem influenciar no aparecimento da PIF são: estresse, susceptibilidade genética, doenças intercorrentes, via de infecção e imunocompetência mediada por células (HOSKINS; LOAR, 1993). A PIF é classificada nas formas efusiva (úmida) e não-efusiva (seca), com base na 
quantidade de derrame cavitário (ascite ou hidrotórax) apresentado pelos animais afetados (ADDIE et al., 2009; DRECHSLER et al., 2011; GELBERG, 2013). Os animais acometidos pela doença podem ser assintomáticos ou apresentar depressão, anorexia e perda de peso, mucosas pálidas, febre e uveíte, embora nenhum sinal clínico seja considerado patognomônico (DRECHSLER et al., 2011; KIPAR et al., 2005). Alguns animais podem apresentar sinais nervosos, tais como: ataxia, nistagmo, incoordenação motora, tremores de intenção, hiperestesia, alterações comportamentais e sinais de alterações nos nervos cranianos; cuja ocorrência depende da área do sistema nervoso afetada (ADDIE; JARRETT, 1998; KIPAR et al., 2005). Histologicamente, a PIF consiste em uma inflamação predominantemente piogranulomatosa localizada ao redor de vasos, principalmente de vênulas (BARKER, 1993; HOSKINS; LOAR, 1993; KIPAR et al., 2005).

Este artigo teve como objetivo descrever um estudo de dez casos da doença, diagnosticados entre 2010-2013 na região sul do Rio Grande do Sul, no Laboratório Regional de Diagnóstico (LRD) da Faculdade de Veterinária da Universidade Federal de Pelotas.

\section{MATERIAL E MÉTODOS}

Os arquivos do LRD entre 2000 e 2013 foram revisados (laudos e protocolos). Foi procedido um inventário sobre os exames histopatológicos e necropsias realizadas em gatos nesse período, de modo a identificar os animais com diagnóstico positivo para PIF. Foram relacionados à idade, sexo, raça, histórico clínico e procedência. Os sinais clínicos, as lesões macro e microscópicas foram revisadas. A doença foi classificada como efusiva ou nãoefusiva de acordo com a presença de derrames cavitários descritos nos arquivos.

\section{RESULTADOS E DISCUSSÃO}

Entre os anos de 2000 e 2013, 318 felinos foram examinados no LRD. Destes, 10 casos $(3,14 \%)$ receberam o diagnóstico positivo para PIF, baseado nas lesões macro e microscópicas, que são consideradas diagnósticas (BARKER, 1993; DRECHSLER et al., 2011; KIPAR et al., 2005; OLIVEIRA et al., 2003). Segundo Gelberg (2013), 12\% das mortes em felinos são associadas à PIF. A idade, o sexo, a raça, a procedência, a forma da doença e os sinais clínicos apresentados pelos gatos afetados constam na Tabela 1. 
Tabela 1 - Casos de Peritonite Infecciosa Felina entre 2000 e 2013, diagnosticados no Laboratório Regional de Diagnóstico da Faculdade de Veterinária da Universidade Federal de Pelotas.

\begin{tabular}{|c|c|c|c|c|c|c|c|c|c|}
\hline \multirow[b]{2}{*}{ № } & \multirow[b]{2}{*}{ Raça } & \multirow[b]{2}{*}{ Idade } & \multirow[b]{2}{*}{ Sexo } & \multirow[b]{2}{*}{ Forma } & \multicolumn{2}{|l|}{ Sinais Clínicos } & \multirow[b]{2}{*}{ Icterícia } & \multirow[b]{2}{*}{ Vômito } & \multirow[b]{2}{*}{ Ascite } \\
\hline & & & & & Emagrecimento & Anorexia & & & \\
\hline 1 & Persa & $3 A$ & $M$ & $E$ & - & - & + & - & + \\
\hline 2 & Siamês & $\begin{array}{c}1 \mathrm{~A} \mathrm{e} \\
6 \mathrm{M}\end{array}$ & $\mathrm{F}$ & $E$ & - & - & - & + & + \\
\hline 3 & Siamês & $6 \mathrm{M}$ & $M$ & $E$ & - & + & + & - & + \\
\hline 4 & SRD & $4 \mathrm{~A}$ & $\mathrm{~F}$ & $E$ & + & + & - & + & + \\
\hline 5 & SRD & N.I. & $\mathrm{F}$ & NE & - & + & + & - & - \\
\hline 6 & Mainecoon & $4 \mathrm{~A}$ & $M$ & $\mathrm{E}$ & + & - & + & - & + \\
\hline 7 & Siamês & $\begin{array}{c}1 \mathrm{~A} \mathrm{e} \\
5 \mathrm{M}\end{array}$ & $M$ & NE & + & + & - & - & - \\
\hline 8 & SRD & $2 \mathrm{~A}$ & $M$ & $E$ & - & - & - & - & + \\
\hline 9 & SRD & $4 \mathrm{~A}$ & M & NE & - & - & + & - & - \\
\hline 10 & SRD & $2 \mathrm{~A}$ & $\mathrm{~F}$ & E & + & + & + & - & + \\
\hline
\end{tabular}

SRD: sem raça definida; N.I.: não informada; A: anos; M: meses; F: fêmea; M: macho; E: efusiva; NE: não efusiva; +: presente; -: ausente.

Neste estudo, a idade dos animais com PIF variou de 6 meses a 4 anos, porém a enfermidade pode ocorrer em gatos com mais de 10 anos, devido ao declínio da resposta imune associado à idade (DRECHSLER et al., 2011; MCREYNOLDS; MACY, 1997). Foi observada uma pequena diferença em relação ao sexo dos animais afetados (6 machos e 4 fêmeas). Os dados de literatura são conflitantes neste aspecto; Rohrbach et al. (2001) mencionam a maior incidência de PIF em machos não castrados enquanto que Foley et al. (1997) e Gelberg (2013) afirmam que não há diferença na distribuição da doença entre os sexos. O maior acesso a ambientes contaminados por parte de gatos machos não castrados é utilizado para justificar, em alguns estudos, a maior incidência nessa categoria.

Dos dez felinos diagnosticados com PIF, cinco foram classificados como sem raça definida e cinco como raça definida, não havendo, portanto, distinção, em relação a essa característica, entre os animais afetados; diferentemente do relatado em alguns estudos, que identificaram uma maior prevalência de PIF em felinos de raça definida (DRECHSLER et al., 2011; MCREYNOLDS; MACY, 1997). Por outro lado, um aumento da susceptibilidade ou diminuição da resistência à PIF ocorre em algumas populações de gatos com alta consanguinidade, fato de ocorrência comum em gatis (OLIVEIRA et al., 2003). Episódios de estresse, como: 
desmame, transporte, cirurgias, doenças intercorrentes e procedimentos veterinários parecem ser importantes no desenvolvimento da PIF. A evolução dos sinais clínicos até a morte dos gatos deste estudo foi de sete a 45 dias. Em geral, a morte sobrevém ao redor de 60 dias após o início dos sinais clínicos, entretanto, isso pode ocorrer entre uma e 12 semanas (McREYNOLDS; MACY, 1997). A taxa de mortalidade de ambas as formas é de aproximadamente 100\% (BARKER, 1993). Em média, 75\% dos casos de PIF são da forma efusiva e ocorrem em gatos com forte resposta imune humoral (DRECHSLER et al., 2011).

Neste estudo, a taxa de prevalência da forma efusiva e não efusiva corresponderam a 70 e $30 \%$, respectivamente. As razões para que ocorra uma determinada forma não estão completamente compreendidas, mas podem estar relacionadas ao tipo principal de célula imune efetora envolvida (GELBERG, 2013). Os principais sinais clínicos descritos foram anorexia e emagrecimento (5/10), icterícia (6/10) e ascite (7/10). Ainda foram relatados vômitos, anemia e hálito amoniacal. Os sinais clínicos observados em seis gatos deste estudo são semelhantes aos descritos na literatura, tanto para a forma efusiva quanto para a forma não-efusiva (ADDIE; JARRETT, 1998; McREYNOLDS; MACY, 1997).

Os achados de necropsia dos gatos com a forma efusiva incluíam peritonite fibrinosa a fibrinopurulenta, ascite, múltiplos nódulos brancacentos na superfície de vísceras como fígado e rins (Figura 1). A efusão cavitária era amarelada ou rósea-avermelhada, translúcida ou levemente opaca, viscosa e, em alguns casos, continham filamentos de fibrina. Quando exposto ao ar, o líquido coagulava. Um exsudato branco-acinzentado recobria todas as superfícies serosas, conferindo-Ihes aspecto granular. Isto era particularmente marcante sobre o fígado, baço e intestinos e, em menor intensidade, sobre o omento, bexiga, parede abdominal, pâncreas e estômago. Em alguns gatos afetados havia derrame semelhante na cavidade torácica. Em gatos com a forma não-efusiva, macroscopicamente, encontravam-se focos inflamatórios granulomatosos multifocais de intensidade variáveis, localizados sob a serosa dos órgãos da cavidade abdominal e que se estendiam ao parênquima subjacente. Essa lesão era mais pronunciada nos rins. Histologicamente, as principais alterações consistiam de graus variáveis de perivasculite e vasculite piogranulomatosas ou não supurativas em vários órgãos, especialmente nos rins e pulmões (Figura 2). Essas alterações 
foram descritas na literatura em associação à forma efusiva da PIF (HOSKINS; LOAR, 1993; McREYNOLDS; MACY, 1997). Além dessas, em casos de PIF foram descritas massas perceptíveis à palpação abdominal, edema escrotal, vários níveis de dispneia com padrão respiratório restrito e ruídos cardíacos abafados em gatos com derrame pleural (ADDIE; JARRETT, 1998; HOSKINS; LOAR, 1993; McREYNOLDS; MACY, 1997). Essas últimas alterações não foram observadas nos gatos deste levantamento, talvez por falta de dados clínicos nos protocolos examinados. Na forma não-efusiva, as manifestações clínicas dependem dos órgãos afetados e geralmente são pouco definidas (ADDIE; JARRETT, 1998; HOSKINS; LOAR, 1993; MCREYNOLDS; MACY, 1997). Gatos com essa forma geralmente apresentam lesões oculares (ADDIE; JARRETT, 1998; MCREYNOLDS; MACY, 1997), principalmente irite, caracterizada por mudança na coloração da íris (ADDIE; JARRETT, 1998; GELBERG, 2013). Nenhum animal deste estudo desenvolveu lesões oculares.

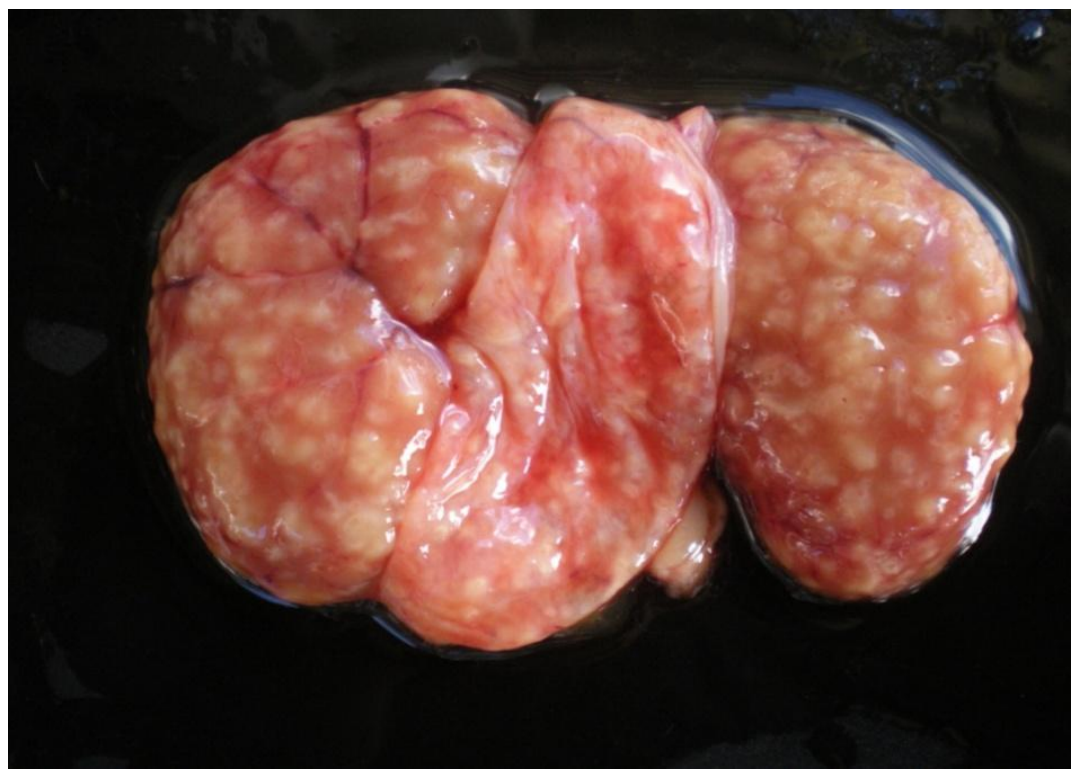

Figura 1 - Rins de felino com a forma efusiva da Peritonite Infecciosa Felina, apresentando superfície capsular com múltiplos nódulos brancacentos. Fonte: Arquivo pessoal dos autores, 2015. 


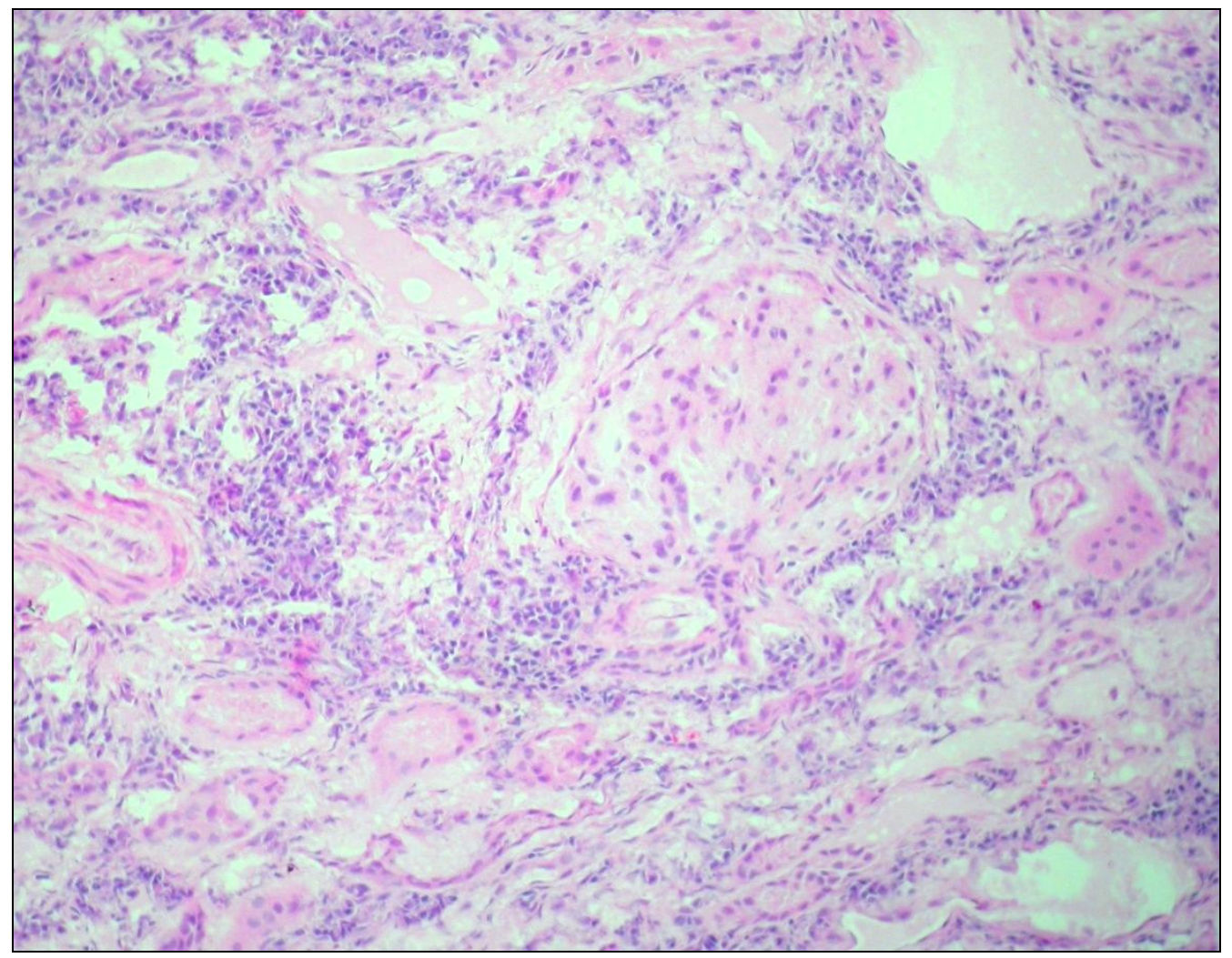

Figura 2 - Infiltrado inflamatório não-supurativo no interstício renal de um felino com a forma efusiva da Peritonite Infecciosa Felina. Fonte: Arquivo pessoal dos autores, 2015.

O diagnóstico da PIF pode ser difícil por causa da variabilidade das manifestações clínicas e do tempo de incubação, mas em muitos casos, pode ser feito através da avaliação do histórico, achados clínicos, exames laboratoriais (sangue e urina), título de anticorpos para coronavírus e exclusão de doenças semelhantes (HOSKINS; LOAR, 1993). A avaliação da efusão, que nos casos positivos para PIF é caracterizada por presença de fibrina e aspecto amarelado e turvo, tem grande importância no diagnóstico presuntivo de PIF (ADDIE; JARRETT, 1998), no entanto, o diagnóstico definitivo é realizado através da necropsia e histopatologia (BARKER, 1993; GELBERG, 2013). Os achados de necropsia e histopatológicos deste estudo estão de acordo com o que foi descrito por Barker (1993), Kipar et al. (2005) e Oliveira et al. (2003) para a doença e foram utilizados para a classificação na forma efusiva e seca.

A forma não-efusiva deve ser diferenciada de linfossarcoma multicêntrico, esteatite, infecções micóticas e toxoplasmose. A forma efusiva da doença deve ser diferenciada de peritonite bacteriana, piotórax (BARKER, 1993), doença cardíaca, neoplasias (linfoma 
hepático, esplênico ou alimentar), doença renal e hepática, pancreatite, esteatite e obstrução da veia cava caudal (ROHRBACH et al., 2011). Não há tratamento eficaz, a maioria das terapias é baseada em cuidados de suporte, incluindo reposição de fluidos e nutrientes. A conduta terapêutica, principalmente, consiste no uso tópico ou sistêmico de corticoides (BRAUND, 1994).

\section{CONCLUSÃO}

Conclui-se que a Peritonite Infecciosa Felina ocorre na zona de abrangência do Laboratório Regional de Diagnóstico, na região sul do Rio Grande do Sul, porém o número real de animais afetados pode ser maior que o descrito neste trabalho, em virtude da confirmação do diagnóstico por necropsia ou histopatologia não ser realizado em todos os casos suspeitos.

\section{A RETROSPECTIVE STUDY OF TEN CASES OF FELINE INFECTIOUS PERITONITIS DIAGNOSED BETWEEN 2000-2013 IN SOUTHERN RIO GRANDE DO SUL}

\section{ABSTRACT}

eline Infectious Peritonitis (FIP) is an immune-mediated viral disease characterized by fibrinousserositis and disseminated vasculitis. The objective of this study was to describe a study of ten cases diagnosed from 2000-2013 in Southern Rio Grande do Sul. During this period, ten out a total of 318 cases (3.14\%) had a diagnosis of FIP. In $70 \%$ of these cases, the animals presented the effusive form of the disease and $30 \%$ non-effusive form. There was no preponderance over the race of affected animals. The age of the cats ranged from six months to four years. The clinical course of the disease to death was between 7-45 days, and clinical signs observed were anorexia, weight loss, jaundice, ascites, vomiting, anemia and ammonia breath. The gross lesions were variable and included peritonitis and whitish foci of serous various viscera in both forms of the disease. Using histopathology, it was observed granulomatous or piogranulomatosas vasculitis and perivasculitis in various organs in both forms. The diagnosis in these cases were made based on clinical, necropsy and histopathology history. We conclude that the feline infectious peritonitis occurs in the region covered by the Regional Diagnostic Laboratory in the Southern Rio Grande do Sul, but the actual number of affected animals may be greater than that described in this study, because the diagnosis confirmed by necropsy or histopathology not be performed in all suspected cases.

Keywords: Cats. Coronavirus. Peritonitis. 


\section{ESTUDIO RETROSPECTIVO DE DIEZ CASOS DE PERITONITIS INFECCIOSA FELINA DIAGNOSTICADOS ENTRE 2000-2013 EN LA REGION SUR DEL RIO GRANDE DEL SUR}

\section{RESUMEN}

a Peritonitis Infecciosa Felina (PIF) es una enfermedad viral inmunomediada, caracterizada por serositis fibrinosa diseminada y vasculitis. El objetivo de este trabajo fue describir un estudio de diez casos de la enfermedad diagnosticados entre 2000 a 2013 en el sur del Rio Grande del Sur. En este período se analizaron materiales de 318 felinos, de los cuales diez casos (3,14\%) fueron diagnosticados con PIF. De estos casos, $70 \%$ de los animales presentó la forma efusiva de la enfermedad y $30 \%$ la forma no efusiva. No hubo preponderancia sobre la raza de los animales afectados. La edad de los gatos varió de seis meses a cuatro años. El curso clínico de la enfermedad hasta la muerte fue entre 7-45 días y los signos clínicos encontrados fueron la anorexia, pérdida de peso, ictericia, ascitis, vómitos, anemia y respiración con olor a amoníaco. Las lesiones macroscópicas fueron variables y incluyeron peritonitis y manchas blanquecinas en la serosa de diversos órganos en ambas formas de la enfermedad. Histológicamente, en ambas formas diagnosticadas, había vasculitis y perivasculitis granulomatosa o piogranulomatosa en varios órganos. EI diagnóstico en los casos analizados fue basado en la historia clínica, la necropsia y histopatología. Se concluye que la Peritonitis Infecciosa Felina ocurre en la región de cobertura del Laboratorio Regional de Diagnóstico, en el sur del Rio Grande del Sur, pero el número real de animales afectados puede ser mayor al que se describe en este trabajo debido a que el diagnóstico fue confirmado por la necropsia o histopatología qué no se lleva a cabo en todos los casos sospechosos.

Palabras clave: Gatos. Coronavirus. Peritonitis.

\section{AGRADECIMENTOS}

Agradecemos à CAPES pelo apoio financeiro.

\section{REFERÊNCIAS}

ADDIE, D. D.; BELAK, S.; BOUCRAUT-BARALON, C.; et al. Feline infectious peritonitis. ABCD guidelines on prevention and management. Journal of Feline Medicine \& Surgery, v. 11, n. 7, p. 594-604, 2009.

ADDIE, D. D.; JARRETT, O. Feline coronavirus infection. In: GREENE, C. E. Infectious Disease Dog and Cat. Athens, Georgia: Saunders, 1998, p. 58-69. 
BARKER, I. K. The peritoneum and retroperitoneum. In: JUBB, K. V. F.; KENNEDY, P. C.; PALMER, N. Pathology of Domestic Animal. San Diego: Academic, 1993, v. 2, p. 425-445.

BRAUND, K. G. Neurological diseases. In: BRAUND, K. G. Clinical Syndrome Veterinary Neurology. St. Louis: Mosby, 1994, p. 81-333.

DRECHSLER, Y.; ALCARAZ, A.; BOSSONG, F. J.; et al. Feline Coronavirus in Multicat Environments. Veterinary Clinics Small Animal, v. 41, p. 1133-1169, 2011.

FOLEY, J. E.; POLAND, A.; CARLSON, J.; PEDERSEN, N. C. Risk factors for feline infectious peritonitis among cats in multiple-cat environments with endemic feline enteric coronavirus. Journal of the American Veterinary Medical Association, v. 210, n. 9, p. 1313-1318, 1997.

GELBERG, H. B. Sistema Alimentar, Peritônio, Omento, Mesentério e Cavidade Peritoneal. In: McGAVIN, M. D.; ZACHARY, J. F. Bases da Patologia em Veterinária, 5. ed. Rio de Janeiro: Elsevier, 2013. Cap. 7, p. 324-406.

HOSKINS, J. D.; LOAR, A. S. Feline infectious diseases. Veterinary Clinics of North America, v. 23, n. 1, p. 2-11, 1993.

KIPAR, A.; MAY, H.; MENGER, S.; et al. Morphologic Features and Development of Granulomatous Vasculitis in Feline Infectious Peritonitis. Veterinary Pathology, v. 42, p. 321-330, 2005.

McREYNOLDS, C.; MACY, D. Feline infectious peritonitis. Part I. Etiology and diagnosis. Compendium on Continuing Education for the Practicing Veterinarian, v. 19, n. 9, p. 1007$1012,1997$.

OLIVEIRA, F. N.; RAFFI, M. B.; SOUZA, T. M.; et al. Peritonite infecciosa felina: 13 casos. Ciência Rural, v. 33, n. 5, p. 905-911, 2003.

ROHRBACH, B. W.; LEGENDRE, A. M.; BALDWIN, C. A.; LEIN, D. H.; REED, W. M.; WILSON, R. $B$. Epidemiology of feline infectious peritonitis among cats examined at veterinary medical teaching hospitals. Journal of the American Veterinary Medical Association, v. 218, n. 7, p. 1111-1115, 2001. 